\title{
15. Anstoß und Motive für das freiwillige Engagement
}

\author{
Doreen Müller, Nicole Hameister \& Katharina Lux
}

\section{Kernaussagen}

Die Hälfte aller Engagierten ist aus eigener Initiative freiwillig tätig geworden, die andere Hälfte wurde angefragt. Besonders häufig nahmen Frauen, 14- bis 29-Jährige und Personen ab 65 Jahren aus eigener Initiative ihre freiwillige Tätigkeit auf.

Die Zahl der aus eigener Initiative Engagierten ist in den letzten fünfzehn Jahren stark angestiegen. Im Jahr 1999 waren es 38,7 Prozent der Engagierten, im Jahr 2014 sind es dagegen bereits 47,2 Prozent.

Direkte soziale Interaktionen sind die häufigsten Anstöße zum Engagement. Die Ansprache durch leitende Personen der Gruppe oder Organisation oder durch Familienmitglieder, Freundinnen und Freunde oder Bekannte werden von fast der Hälfte der Engagierten als ausschlaggebend für die Aufnahme ihres Engagements genannt.

Informations- und Kontaktstellen werden im Trendvergleich wichtiger. Im Vergleich zu 1999 werden diese Einrichtungen 2014 wesentlich häufiger von den Engagierten als Anstoß genannt.

Männer werden häufiger durch leitende Personen von Organisationen geworben als Frauen. Frauen haben hingegen anteilig häufiger durch Erfahrungen in der Familie zu ihrem Engagement gefunden als Männer.

Die Motive der Engagierten sind vielfältig, der Spaß steht dabei an erster Stelle. Es engagieren sich ebenfalls viele, um mit anderen zusammenzukommen - also aus sozialen Motiven. Seltener ist das Engagement motiviert durch den Wunsch, Qualifikationen zu erwerben.

Soziale, vor allem auf zwischenmenschliche Kontakte bezogene Motive werden eher von Frauen und Älteren genannt. Motive, die sich auf den eigenen Nutzen richten, sind hingegen eher für Männer und jüngere Personen wichtig.

\subsection{Einleitung}

Warum engagieren sich Menschen, und was hat sie veranlasst, ihre freiwillige Tätigkeit aufzunehmen? Zum einen sind äußere Faktoren relevant, denn manche Personen haben eher die Gelegenheiten und die Ressourcen, sich zu engagieren, als andere. So wurde bereits gezeigt, dass Geschlecht,
Alter und Bildung (siehe Kapitel 3) sowie das soziale und kulturelle Umfeld eines Menschen einen großen Einfluss haben (vgl. Wilson \& $\mathrm{Mu}-$ sick 1998). Daneben gibt es jedoch auch meist einen konkreten Anstoß sowie Motive auf der individuellen Ebene, die freiwilliges Engagement

(C) Der/die Autor(en) 2017

J. Simonson et al. (Hrsg.), Freiwilliges Engagement in

Deutschland, Empirische Studien zum bürgerschaftlichen

Engagement, DOI 10.1007/978-3-658-12644-5_16 
begünstigen. Viele freiwillige Tätigkeiten ergeben sich zum Beispiel aus den Anforderungen des Alltags: Die Basketballspielerin wird gefragt, ob sie ehrenamtlich Jugendtrainerin werden und anderen ihren Spaß an dem Sport vermitteln möchte. Eltern engagieren sich im Schulförderverein, um bessere Lernbedingungen zu schaffen. Aus dem Protest an einer Baumaßnahme in der Nachbarschaft wird eine Bürgerinitiative. Andere Menschen wenden sich an Freiwilligenagenturen, weil sie ihre Zeit für etwas Gemeinnütziges spenden möchten und noch keine konkreten Ideen haben, wofür. Bei all diesen Engagierten werden unterschiedliche Anlässe und Motive wirksam, die zur Aufnahme und zum Beibehalten ihrer freiwilligen Tätigkeit führen.

In diesem Kapitel wird der Frage nachgegangen, was die Engagierten zur Aufnahme eines freiwilligen Engagements bewegt hat. Dazu werden folgende Aspekte untersucht: Wurde das Engagement aus eigener Initiative aufgenommen oder wurden die Engagierten gefragt beziehungsweise angesprochen? Aus welchem Umfeld kam der direkte Anstoß zum Engagement? Welche Motive bewegen die Engagierten zu ihrer freiwilligen Tätigkeit?

Eigeninitiative und Ansprache durch Andere: Einige Engagierte wurden von allein aktiv, engagieren sich also aus eigener Initiative. Andere wurden persönlich gefragt und nahmen daraufhin eine freiwillige Tätigkeit auf. Hier sind Unterschiede in den verschiedenen Bevölkerungsgruppen denkbar: Werden eher Frauen oder eher Männer gefragt? Hängt Eigeninitiative im freiwilligen Engagement auch vom Alter oder dem Bildungsniveau ab? Die Antworten dazu können Hinweise darauf geben, auf welchen Wegen welche Bevölkerungsgruppen am ehesten $\mathrm{zu}$ ihrem Engagement finden und welche Zugangswege gestärkt werden könnten. Insgesamt ist die Frage nach der Eigeninitiative eng verbunden mit dem konkreten Anstoß zum Engagement.

Anstoß zum Engagement: Anstöße zum Engagement können bestimmte Erfahrungen, Situationen im Leben und Informationen sein, die im Rückblick betrachtet dazu führten, dass eine Person ein Engagement aufgenommen hat. Anstöße können auch von bereits engagierten Personen aus dem familiären oder beruflichen Umfeld ausgehen. Auch eine positive Erfahrung kann Engagement auslösen: Viele Menschen bleiben zum Beispiel nach einem Freiwilligen Sozialen Jahr freiwillig engagiert (siehe Kapitel 5). Zudem sind öffentliche Aufrufe zum Engagement, etwa über das Fernsehen, Plakatwerbung und das Internet denkbar. Es ist zu vermuten, dass Anstöße aus dem direkten Umfeld einer Person einen stärkeren Appell-Charakter besitzen und eher zu Engagement führen als zum Beispiel unpersönliche Aufrufe zum Engagement über Fernsehen und Radio.

Die Zugehörigkeit zu einer bestimmten Bevölkerungsgruppe hinsichtlich des Geschlechts, des Alters oder der Bildung kann auch hier eine wichtige Rolle spielen. Diese Zugehörigkeiten bestimmen die persönlichen Ressourcen, das soziale Umfeld und viele andere Aspekte im Leben eines Menschen. Junge Menschen verfolgen oft andere Ziele im Leben als Ältere, sie suchen vielleicht eher nach Orientierungshilfen beispielsweise in der Familie oder im Sportverein. Frauen sind auch heute noch umfassender in familiale Aufgaben eingebunden als Männer; ihr Umfeld setzt sich tendenziell aus anderen Personen zusammen und sie werden mit anderen Themen konfrontiert. Ähnlich verhält es sich mit den Bildungsgruppen. Menschen höherer Bildung bewegen sich oft in anderen Lebenskontexten als Menschen niedriger Bildung. Daher ist auch hier $\mathrm{zu}$ erwarten, dass unterschiedliche Anstöße für die verschiedenen Bildungsgruppen bedeutsam sind.

Motive für Engagement: Die von außen kommenden Anstöße müssen jedoch auch auf eine individuelle innere Motivlage treffen, die die Bereitschaft $\mathrm{zu}$ freiwilligem Engagement begünstigt. In der Literatur werden verschiedene Möglichkeiten diskutiert, Motive zu kategorisieren. Dazu zählt die Einordnung in altruistische und egoistische Motive, die das Handeln für den Nutzen anderer vom Handeln für einen persönlichen Nutzen unterscheidet (Bierhoff, Schülken 
\& Hoof 2007; Moschner 2002). Eine zweite Kategorisierung ist die Einteilung in intrinsische versus extrinsische Motivation, die sich darauf bezieht, ob die Motivation aus dem Selbst heraus oder von außen in Form einer Belohnung kommt. Im Zusammenhang mit freiwilligem Engagement findet sich jedoch häufig der Hinweis auf die Vielfältigkeit der Motive. Viele Menschen sind nicht nur engagiert, weil sie anderen helfen möchten, sondern auch, weil ihnen die Tätigkeit Freude bereitet, weil sie Menschen treffen können, weil sie Qualifikationen erwerben möchten (Haumann 2014). Daher werden die Motive für Engagement häufig auch als ,Motivbündel' bezeichnet (Hacket \& Mutz 2002) und können am besten gemeinsam und ohne die Aufspaltung in abstrakte Kategorien betrachtet werden (Braun \& Bischoff 1999).

Auch im Freiwilligensurvey 2014 werden verschiedene Arten von Motiven für das Engagement erfasst und gemeinsam ausgewertet: Der Einsatz für Hilfebedürftige kann gleichzeitig den persönlichen Wunsch, die Gesellschaft mitzugestalten, befriedigen. Wenn eine freiwillige Tätigkeit keine Freude mehr bereitet, wird es für die Engagierten sicher schwierig, allein aus Pflichtgefühl dabeizubleiben. Engagement kann außerdem dazu beitragen, dass Menschen sich besser fühlen (siehe Kapitel 17) - der Spaß an der Sache ist dabei ein wichtiger Faktor. Ebenfalls eine Rolle spielen mag, dass ein bestimmtes Engagement im Umfeld zu Anerkennung führt und für den Berufsweg wichtige Erfahrungen gemacht werden können. In einigen wenigen Fällen bleiben Engagierte möglicherweise aufgrund einer Aufwandsentschädigung motivierter dabei (allerdings erhalten nur sehr wenige Engagierte Geldzahlungen, siehe Kapitel 14).

Diese Aspekte können aber, abhängig von der individuellen Situation der Engagierten, unterschiedliches Gewicht für den Einzelnen haben. So sind wie bei den Anstößen auch hinsichtlich der Motivation für freiwilliges Engagement Unterschiede zwischen verschiedenen Bevölkerungsgruppen $\mathrm{zu}$ erwarten. Manche Studien zeigten zum Beispiel, dass Frauen sich eher aus sozialen Gründen engagieren, zum Beispiel um anderen zu helfen (Clary, Snyder, Ridge, Copeland, Stukas, Haugen \& Miene 1998). In anderen Studien hingegen gibt es kaum Unterschiede zwischen den Geschlechtern (Burns, Reid, Toncar, Anderson \& Wells 2008). Auch ältere Menschen engagieren sich oft aus anderen Gründen als Jüngere. Junge Menschen möchten zum Beispiel eher Erfahrungen sammeln und dabei insbesondere Qualifikationen erwerben, die auf dem Arbeitsmarkt nützlich sind. Für die jüngsten Engagierten und die ältesten Engagierten sind jedoch auch die sozialen Kontakte beim Engagement von großer Bedeutung (HaskiLeventhal, Ronel, York \& Ben-David 2008; Braun \& Bischoff 1999). Dass die Motive für Engagement durch die sozioökonomischen Merkmale beeinflusst werden, zeigt sich auch bei unterschiedlich gebildeten Gruppen. Insbesondere hochgebildete Personen trauen sich zum Beispiel zu, mit ihrem Engagement die Gesellschaft mitgestalten zu können (Böhnke \& Dathe 2010). Für alle Gruppen gilt also, dass komplexe Motivbündel Menschen dazu bewegen sich zu engagieren und Selbstlosigkeit und Eigennutz sich nicht ausschließen, sondern sinngebend miteinander verknüpft werden (Deutscher Bundestag 2012; Haumann 2014; Hacket \& Mutz 2002).

Die Forschungsfragen, die in diesem Kapitel beantwortet werden sollen, lauten:

1. Haben die Engagierten ihr Engagement häufiger aus eigener Initiative heraus aufgenommen oder wurden sie angesprochen?

2. Gibt es Unterschiede zwischen den Geschlechtern, den Alters- und den Bildungsgruppen hinsichtlich der Eigeninitiative?

3. Welche Anstöße nennen Engagierte? Werden direkte Anstöße aus dem näheren Umfeld häufiger genannt als indirekte Aufrufe?

4. Welche Geschlechter-, Alters- und Bildungsunterschiede finden sich hinsichtlich der Anstöße? 
5. Welche persönlichen Motive sind für die Engagierten am wichtigsten?

\subsection{Datengrundlage}

Die in diesem Kapitel berichteten Befunde basieren auf gewichteten Analysen. Für die Gewichtung wurden in allen Erhebungsjahren dieselben Gewichtungsmerkmale berücksichtigt. Dabei handelt es sich um die Designgewichtung, die sich auf die Auswahlwahrscheinlichkeit einer Person bezieht (Haushaltsgröße, Zahl der Festnetznummern und, nur für das Jahr 2014, auch die Zahl der Mobilfunknummern) und Gewichtungsmerkmale, die Abweichungen der Stichprobenverteilung von der Grundgesamtheit in bestimmten Dimensionen ausgleichen (Bundesland, Gemeindegrößenklassen, Geschlecht, Altersgruppen). Von einer Hinzunahme weiterer Gewichtungsmerkmale wie etwa des Bildungsstandes haben wir abgesehen, um die Vergleichbarkeit mit den bisher veröffentlichten Berichten zu erhalten.

Eigeninitiative: Bezüglich der Initiative wurden die Engagierten Folgendes zu ihrer freiwilligen Tätigkeit gefragt (bei Engagierten mit mehreren Tätigkeiten ist das die zeitaufwändigste Tätigkeit, bei Personen mit einer freiwilligen Tätigkeit ihre einzige):

Ging die Initiative von Ihnen selbst aus oder wurden Sie gefragt, ob Sie die Aufgaben übernehmen wollen?

Hierbei mussten sich die Engagierten für eine der beiden Antwortmöglichkeiten entscheiden. Diese Frage wurde seit 1999 in jeder Welle des
6. Welche Geschlechter-, Alters- und Bildungsunterschiede können bezüglich der Motive berichtet werden?

Freiwilligensurveys gestellt und lässt sich damit im Zeitverlauf auswerten.

Anstöße zum Engagement: Weiterhin konnten die Engagierten konkrete Anstöße für ihre freiwillige Tätigkeit im Rahmen einer Mehrfachnennung angeben. Die Frage lautet:

Woher kam für Sie damals der Anstoß, die Tätigkeit zu übernehmen? Ich lese Ihnen nun verschiedene Möglichkeiten vor. Sagen Sie mir bitte jeweils, ob die Aussage zutrifft oder nicht zutrifft. Der Anstoß kam von...

Die möglichen Anstöße zum Engagement (siehe Tabelle 15-1) lassen sich thematisch nach dem sozialen Umfeld, aus dem sie kamen, einordnen. Folgende vier Bereiche lassen sich dabei differenzieren: 1) das Engagementumfeld: Hier sind Einflüsse gemeint, die direkt aus der Gruppe oder Organisation der Engagierten stammen; 2) das private Umfeld bezieht sich auf Anstöße aus dem Familien-, Freundes- oder Bekanntenkreis; 3) das berufliche Umfeld meint Anstöße aus der schulischen oder beruflichen Qualifikations- sowie Arbeitsphase; sowie 4) Anstöße außerhalb des direkten Umfelds, also zum Beispiel Informationsstellen oder Berichte im Fernsehen. Diese Einordnung wird in Tabelle 15-1 dargestellt. Einige der Aussagen wurden bereits seit 1999 in gleicher Weise erfasst und sind daher im Trendvergleich analysierbar. 
Tabelle 15-1: Einordnung der Anstöße zum Engagement

\begin{tabular}{|c|c|c|}
\hline Einordnung & Anstöße & $\begin{array}{l}\text { Trendvergleich } \\
\text { möglich zu ... }\end{array}$ \\
\hline Engagementumfeld & ... Leitenden Personen aus Ihrer Gruppe oder Organisation & allen Wellen \\
\hline \multirow[t]{2}{*}{ Privates Umfeld } & $\begin{array}{l}\text {... Familienmitgliedern, Freunden oder Bekannten, die dort } \\
\text { schon aktiv waren }\end{array}$ & / \\
\hline & ... Erfahrungen in der Familie & / \\
\hline \multirow[t]{4}{*}{ Berufliches Umfeld } & ... Ihrer Tätigkeit während des Freiwilligendienstes a & / \\
\hline & ... Ihrer Schule, Hochschule oder Ihrer Ausbildung & / \\
\hline & ... Ihrer Tätigkeit während des Zivildienstes b & / \\
\hline & ... Ihrem Arbeitgeber ${ }^{c}$ & / \\
\hline \multirow{2}{*}{$\begin{array}{l}\text { Außerhalb des direkten } \\
\text { Umfelds }\end{array}$} & ... Einer Informations- und Kontaktstelle & allen Wellen \\
\hline & ... Hinweisen aus den Medien oder dem Internet & / \\
\hline
\end{tabular}

Quelle: FWS 1999, FWS 2004, FWS 2004, FWS 2014. Eigene Darstellung (DZA).

Anmerkungen: ${ }^{a}$ Nur Personen, die einen Freiwilligendienst ausgeübt haben oder ausüben (4,5 Prozent aller Engagierten beantworteten diese Frage), ${ }^{b}$ Nur Personen, die einen Zivildienst ausgeübt haben oder ausüben (5,0 Prozent aller Engagierten beantworteten diese Frage), 'Nur Personen, die erwerbstätig waren oder sind (84,7 Prozent aller Engagierten beantworteten diese Frage).

Motive für Engagement: Die Engagierten wurden nach ihren Motiven für ihr gesamtes Engagement, nicht nur auf ihre zeitaufwändigste Tätigkeit bezogen, befragt:

Sagen Sie mir bitte, ob Sie den folgenden Aussagen über Ihr Engagement voll und ganz zustimmen, eher zustimmen, teils/teils zustimmen, eher nicht zustimmen oder ganz und gar nicht zustimmen.

Die Motive, die zur Auswahl standen, stellen verschiedene Ausrichtungen der Motivation dar: Es wurden soziale Aspekte erfasst, die sich vor allem auf das eigene Wohlbefinden und das soziale Miteinander beziehen sowie Motive, die eher einen persönlichen immateriellen oder materiellen Gewinn, zum Beispiel den beruflichen oder finanziellen Nutzen, erfassen. Mögliche Motive waren:

- Mein Engagement macht mir Spaß.

- Ich will vor allem mit anderen Menschen zusammenkommen.

- Ich will die Gesellschaft zumindest im Kleinen mitgestalten.

- Ich möchte mit Menschen anderer Generationen zusammensein.

- Ich will mir Qualifikationen erwerben, die im Leben wichtig sind.

- Ich will Ansehen und Einfluss in meinem Lebensumfeld gewinnen.

- Ich will durch mein Engagement auch beruflich vorankommen.

- Ich möchte mir durch das Engagement etwas dazuverdienen. 


\subsection{Eigeninitiative und Ansprache durch Andere}

Knapp die Hälfte der Engagierten, insgesamt 47,2 Prozent, ist durch die eigene Initiative freiwillig tätig, dagegen wurden mit 52,8 Prozent über die Hälfte der Engagierten gefragt, ob sie die freiwillige Tätigkeit übernehmen wollen (Abbildung 15-1). Besonders bei Frauen und jungen Menschen, also 14- bis 29-Jährigen, ist der Anteil der aus eigener Initiative Engagierten hoch.

\section{Abbildung 15-1: Anteile freiwillig Engagierter, die sich aus eigener Initiative heraus engagieren oder gefragt wurden, 2014, a) gesamt, nach Geschlecht und Alter, b) nach Bildung}

a) gesamt, nach Geschlecht und Alter

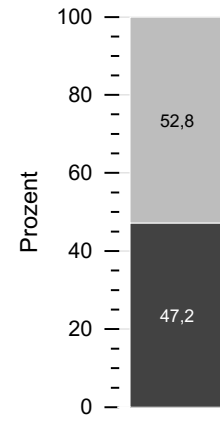

Gesamt

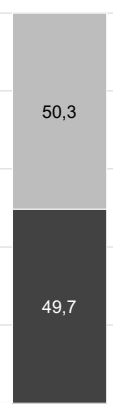

Frauen

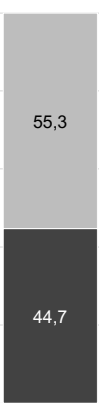

Männer

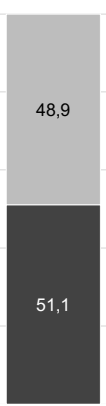

14-29 Jahre
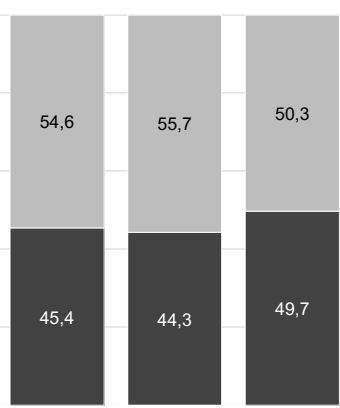

30-49

Jahre

50-64 65 Jahre

Jahre und älter

b) nach Bildung

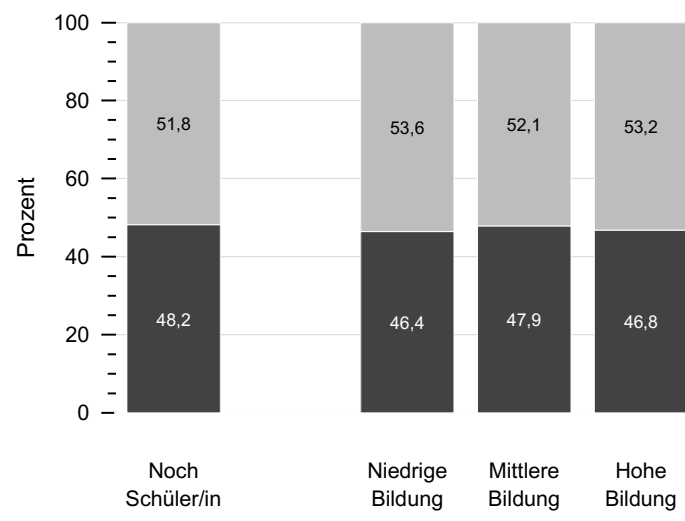

Quelle: FWS 2014, gewichtet, eigene Berechnungen (DZA). Basis: Alle Engagierten ( $n=12.318-12.321)$.

Personen unter 30 Jahren wurden sogar etwas häufiger aus Eigeninitiative heraus freiwillig tätig als dass sie gefragt wurden, Aufgaben zu übernehmen. Im Vergleich zu den 30- bis 64-jährigen
Engagierten geben vor allem engagierte Personen ab 65 Jahren an, sich aus eigener Initiative zu engagieren. Unterschiede hinsichtlich der Bildung lassen sich nicht feststellen, Personen aus 
allen Bildungsgruppen wurden etwas häufiger gefragt als aus eigener Initiative freiwillig tätig. Insgesamt sind die Unterschiede jedoch gering.

Im Zeitvergleich zeigt sich ein steigender Trend hin zu mehr Eigeninitiative (Abbildung 15-2). Im Jahr 1999 wurden insgesamt 38,7 Pro- zent der Engagierten aus Eigeninitiative tätig im Vergleich zu 58,1 Prozent, die von anderen gefragt wurden. Fünfzehn Jahre später sind 47,2 Prozent aus Eigeninitiative tätig gewordenen und 52,8 Prozent der Engagierten wurden gefragt.

\section{Abbildung 15-2: Anteile freiwillig Engagierter, die sich aus eigener Initiative heraus engagieren oder gefragt wurden, im Zeitvergleich}

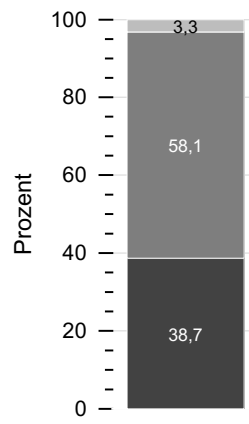

1999

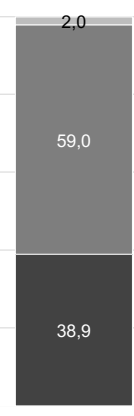

2004

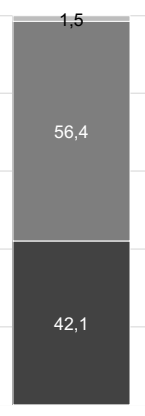

2009

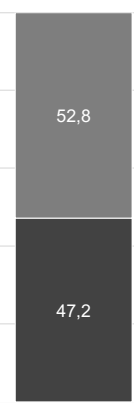

2014

Quelle: FWS, gewichtet, eigene Berechnungen (DZA). Basis: Alle Engagierten. FWS 1999 ( $n=4.654)$, FWS 2004

$(n=5.121)$, FWS $2009(n=6.973)$, FWS $2014(n=12.321)$.

\subsection{Anstöße zum Engagement}

Mit 48,0 Prozent gibt fast die Hälfte der Engagierten an, dass leitende Personen aus der Gruppe oder Organisation den konkreten Anstoß zum Engagement gegeben haben (Abbildung 15-3). Dies ist damit der anteilig am häufigsten genannte Anstoß. Über bereits engagierte Familienmitglieder, Freundinnen und Freunde oder Bekannte kamen insgesamt 44,9 Prozent zu ihrer freiwilligen Tätigkeit. Nicht nur Personen aus dem familiären Umfeld, sondern auch Erfahrungen, die innerhalb der Familie gemacht werden, können Anlass sein sich zu engagieren. Insgesamt 27,8 Prozent geben dies als Anstoß an.

Von den möglichen Anstößen aus dem beruflichen Umfeld wird am häufigsten die Schule, Hochschule oder Ausbildung mit 14,3 Prozent genannt. Im Freiwilligensurvey 2014 wurden zu einigen Anstößen nur Personen befragt, für die dieser Anstoß relevant ist: Ob der Arbeitgeber das Engagement angestoßen hat, wurden nur aktuell erwerbstätige Engagierte gefragt; ob der Freiwilligendienst eine Rolle spielte, wurden nur Personen gefragt, die aktuell einen Freiwilligendienst leisten oder früher einen geleistet haben; und ob das Engagement durch den Zivildienst angestoßen wurde, wurden nur Engagierte, die in der Vergangenheit einen Zivildienst geleistet haben, gefragt. Um eine Vergleichbarkeit zu den anderen Anstößen herzustellen, wurden die Antworten auf alle Engagierten hochgerechnet. Dass ihr Arbeitgeber den Anstoß geliefert hat, sagten 5,8 Prozent aller Engagierten, also weniger als 
ein Zehntel derjenigen, die zurzeit sowohl engagiert als auch erwerbstätig sind. Den Freiwilligendienst als ausschlaggebend benannten 1,0 Prozent, was knapp ein Viertel der Engagierten ist, die einmal einen Freiwilligendienst geleistet haben oder aktuell leisten (siehe Kapitel 5). Den Zivildienst benannten 0,4 Prozent aller Engagierten als Anstoß, das heißt weniger als ein Zehntel der Engagierten, die früher einen Zivil- dienst ausgeübt haben oder derzeit einen solchen ausüben.

Dass der Anstoß von außerhalb des direkten sozialen Umfeldes über eine Informations- und Kontaktstelle oder durch Hinweise aus den Medien oder dem Internet kam, wird von je knapp einem Zehntel der Engagierten berichtet (9,5 Prozent beziehungsweise 8,5 Prozent).

\section{Abbildung 15-3: Angaben freiwillig Engagierter zu den Anstößen zu ihrem Engagement 2014}

\section{Engagementumfeld \\ Leitende Personen aus Engagementumfeld}

Privates Umfeld

Mitglieder der Familie, Freunde oder Bekannte Erfahrungen in der Familie

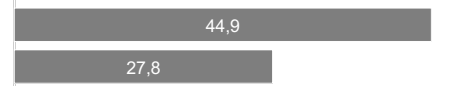

Berufliches Umfeld Schule, Hochschule, Ausbildung Arbeitgeber* Freiwilligendienst* Zivildienst*

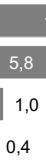

1,0

0,4

Außerhalb des direkten Umfelds

Informations- und Kontaktstelle

Medien oder Internet

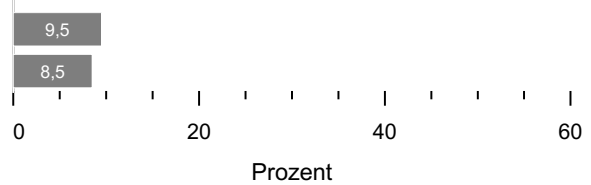

Quelle: FWS 2014, gewichtet, eigene Berechnungen (DZA). Basis: Alle Engagierten. $(n=12.516)$.

* Die Frage nach dem Arbeitgeber beantworteten nur Erwerbstätige (84,7 Prozent der Engagierten), die Frage nach dem Freiwilligendienst nur Personen, die aktuell einen Freiwilligendienst leisten oder früher einen geleistet haben (4,5 Prozent der Engagierten) und die Frage nach dem Zivildienst nur Männer, die früher einen Zivildienst geleistet haben (5,0 Prozent der Engagierten). 
Männer geben anteilig häufiger als Frauen an, direkt im Engagementumfeld von leitenden Personen angesprochen worden zu sein (Abbildung 15-4). Das entspricht auch den Erkenntnissen aus der Frage zur Eigeninitiative - mehr Männer als Frauen wurden von Personen aus der Gruppe oder Organisation gefragt, ob sie ein Engagement übernehmen würden. Während bei Frauen konkrete Erfahrungen in der Familie häufiger einen Anstoß als bei Männern liefern, ist das Ge- schlechterverhältnis beim Anstoß durch bereits engagierte Personen des unmittelbaren Umfelds in Familie, Freundes- und Bekanntenkreis ausgewogen. Bei den Fragen zum beruflichen Umfeld gibt es kaum Unterschiede zwischen Frauen und Männern, abgesehen vom Zivildienst. Durch externe Anstöße in Informations- und Kontaktstellen sowie in Medien und Internet fanden etwas mehr Frauen als Männer zu ihrer freiwilligen Tätigkeit.

\section{Abbildung 15-4: Angaben freiwillig Engagierter zu den Anstößen zu ihrem Engagement 2014, nach Geschlecht}

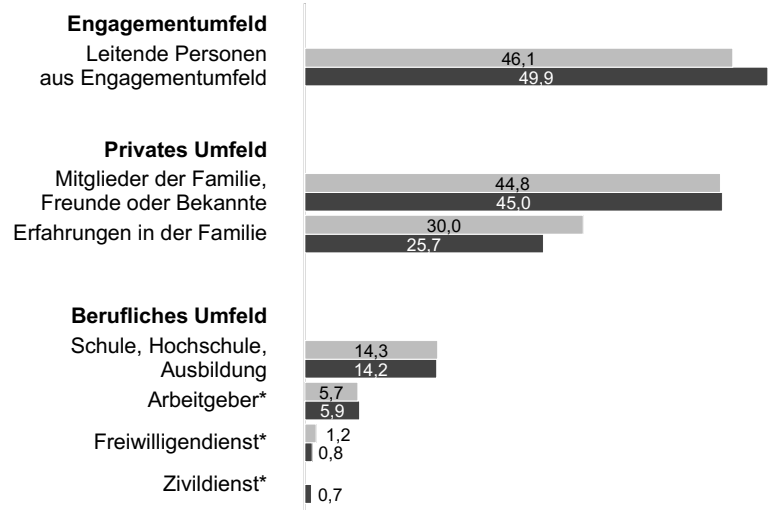

Außerhalb des direkten Umfelds

Informations- und
Kontaktstelle
Medien oder Internet

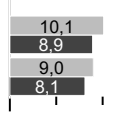

0

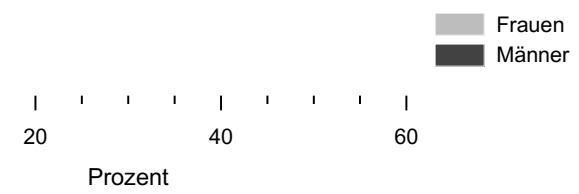

Prozent

Quelle: FWS 2014, gewichtet, eigene Berechnungen (DZA). Basis: Alle Engagierten ( $n=12.516)$.

* Die Frage nach dem Arbeitgeber beantworteten nur Erwerbstätige (84,7 Prozent der Engagierten), die Frage nach dem Freiwilligendienst nur Personen, die aktuell einen Freiwilligendienst leisten oder früher einen geleistet haben (4,5 Prozent der Engagierten) und die Frage nach dem Zivildienst nur Männer, die früher einen Zivildienst geleistet haben (5,0 Prozent der Engagierten). 
Erfahrungen in der Familie als Anstoß für Engagement sind über die Altersgruppen hinweg ebenso ähnlich wie bei der Verteilung über die Geschlechter. Bei den anderen möglichen Anstößen sind die Altersgruppenunterschiede ausgeprägter (Abbildung 15-5). Im Engagementumfeld zeigt sich, dass dieses bis zur Altersgruppe der 50- bis 64-Jährigen weniger wichtig wird, danach aber wieder größere Bedeutung für den Engagementanstoß gewinnt. Den deutlichsten Altersgruppenunterschied findet man im privaten Umfeld: Dass der Zugang zum Engagement über bereits engagierte Familienmitglieder, Freundinnen und Freunde oder Bekannte erfolgte, nennt am häufigsten die jüngste Gruppe der 14- bis 29-Jährigen und am seltensten die älteste Altersgruppe ab 65 Jahren. Hier könnte eine Rolle spielen, dass Ältere insgesamt weniger häufig engagiert sind als Jüngere (siehe Kapitel 3). Da beispielsweise Freundschaften oft innerhalb der eigenen Altersgruppe eingegangen werden, kann man davon ausgehen, dass sich aus dem Freundeskreis älterer Menschen vermutlich weniger Personen engagieren als aus dem von jüngeren Personen. Somit fällt das soziale Umfeld als bedeutsamer Anstoß zum Engagement für Ältere weniger ins Gewicht. In Bezug auf Erfahrungen in der Familie, die zu eigenem Engagement anregen, zeigen sich hingegen keine bedeutsamen Unterschiede in Bezug auf das Alter. Bei den anderen Anstößen zeigen sich deutliche Unterschiede zwischen der jüngsten und ältesten gegenüber den mittleren Altersgruppen: Das trifft beispielsweise auf Anstöße aus dem beruflichen Umfeld zu, aber auch hinsichtlich der externen Anstöße durch Informations- oder Kontaktstellen sowie durch Medien und Internet. 
Abbildung 15-5: Angaben freiwillig Engagierter zu den Anstößen zu ihrem Engagement 2014, nach Alter

Engagementumfeld

Leitende Personen aus Engagementumfeld

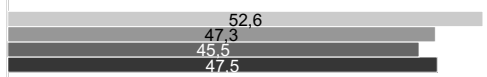

\section{Privates Umfeld}

Mitglieder der Familie, Freunde oder Bekannte

Erfahrungen in der Familie

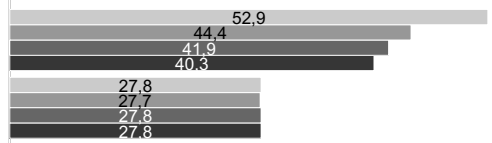

Berufliches Umfeld

Schule, Hochschule, Ausbildung

Arbeitgeber*

Freiwilligendienst*

Zivildienst* $^{*}$

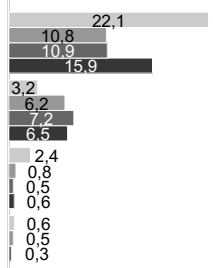

Außerhalb des direkten Umfelds

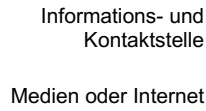

Medien oder Internet

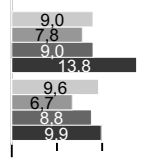

0

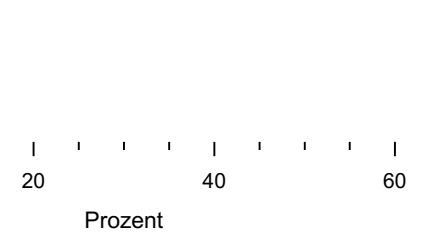

14-29 Jahre

30-49 Jahre

50-64 Jahre

65 Jahre und älter

Quelle: FWS 2014, gewichtet, eigene Berechnungen (DZA). Basis: Alle Engagierten $(n=12.516)$.

* Die Frage nach dem Arbeitgeber beantworteten nur Erwerbstätige (84,7 Prozent der Engagierten), die Frage nach dem Freiwilligendienst nur Personen, die aktuell einen Freiwilligendienst leisten oder früher einen geleistet haben (4,5 Prozent der Engagierten) und die Frage nach dem Zivildienst nur Männer, die früher einen Zivildienst geleistet haben (5,0 Prozent der Engagierten). 
Unterschiede zwischen den Bildungsgruppen zeigen sich zum einen beim Anstoß durch leitende Personen aus dem Engagementumfeld (Abbildung 15-6). Vor allem Schülerinnen und Schüler geben dies als Anstoß an, und am wenigsten häufig wird dieser Anstoß von Personen mittlerer Bildung genannt. Zum anderen zeigen sich deutliche Unterschiede im Bereich des privaten Umfelds, das bei den niedrigeren Schulabschlüssen den größten Einfluss hat. Im berufli- chen Umfeld findet sich vor allem beim Anstoß durch die Schule, Hochschule oder Ausbildung ein tendenziell umgekehrtes Muster: Je höher die abgeschlossene Schulbildung, umso eher wurde im Rahmen von Schul- oder Berufsbildung der Anstoß zum Engagement gefunden. Am bedeutsamsten ist die Ausbildungsstätte hingegen für Personen, die noch zur Schule gehen, was zeigt, dass die Schule für sie ein wesentlicher Lebensbereich ist, der sich auch im Engagement auswirkt. 
Abbildung 15-6: Angaben freiwillig Engagierter zu den Anstößen zu ihrem Engagement 2014, nach Bildung

Engagementumfeld

Leitende Personen aus Engagementumfeld

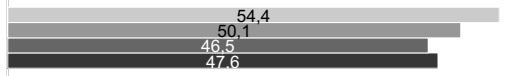

Privates Umfeld

Mitglieder der Familie, Freunde oder Bekannte

Erfahrungen in der Familie

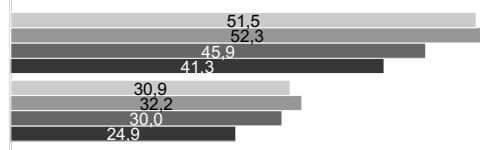

Berufliches Umfeld

Schule, Hochschule, Ausbildung

Arbeitgeber*

Freiwilligendienst*

Zivildienst $^{*}$

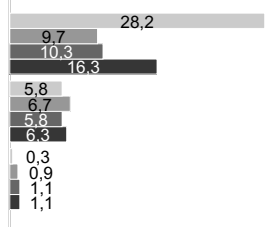

$\mid \begin{aligned} & 0,2 \\ & 0,3 \\ & 0,5\end{aligned}$

Außerhalb des direkten Umfelds

$$
\begin{array}{r}
\text { Informations- und } \\
\text { Kontaktstelle }
\end{array}
$$

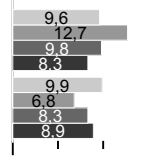

0

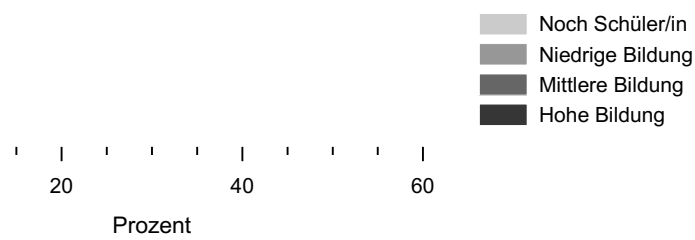

Prozent

Quelle: FWS 2014, gewichtet, eigene Berechnungen (DZA). Basis: Alle Engagierten ( $n=12.512$ ).

* Aufgrund von Filterführungen beantworteten die Frage nach dem Arbeitgeber 84,7 Prozent, die Frage nach dem Freiwilligendienst 4,5 Prozent und die Frage nach dem Zivildienst 5,0 Prozent der Engagierten. 
Im Trend können die beiden Kategorien ,Leitende Personen' aus der Organisation und ,Informations- oder Kontaktstelle betrachtet werden, diese wurden seit 1999 durchgängig und mit gleicher Formulierung erfasst (Abbildung 15-7). Hinsichtlich des Anstoßes durch leitende Personen aus der Gruppe oder Organisation setzt sich der Anstieg, der zwischen 1999 und 2009 zu beobachten war, nicht fort, bleibt aber nah am Wert aus dem Jahr 2009. Seit 2009 gibt etwa die Hälfte der Engagierten an, dass sie direkt angesprochen wurden und daraufhin ihre freiwillige Tätigkeit aufgenommen haben. Vereine, Verbände und andere Organisationen sowie die Personen, die dort Leitungsfunktionen innehaben, sind also wichtige Initiatoren für Engagement. Der leichte Rückgang im Jahr 2014 kann möglicherweise auch dem Wandel des Engagements hin zu mehr informell organisierten Tätigkeiten geschuldet sein. Dennoch bleibt der persönliche Kontakt sehr wichtig für Organisationen, um Freiwillige zu rekrutieren.

Auf der anderen Seite wird der Anstoß durch eine Informations- oder Kontaktstelle seit 1999 kontinuierlich über die Wellen hinweg immer häufiger genannt und liegt nun bei einem knappen Zehntel der Engagierten. Die gestiegene Bedeutung von Informationsstellen kann darauf zurückzuführen sein, dass es heute mehr Informations- und Kontaktstellen gibt als noch vor fünfzehn Jahren. So können Personen, die sich engagieren möchten, diese Stellen stärker wahrnehmen und sie eher in Anspruch nehmen, um eine passende freiwillige Tätigkeit zu finden.

\section{Abbildung 15-7: Angaben freiwillig Engagierter zu den Anstößen zu ihrem Engagement im Zeitvergleich}

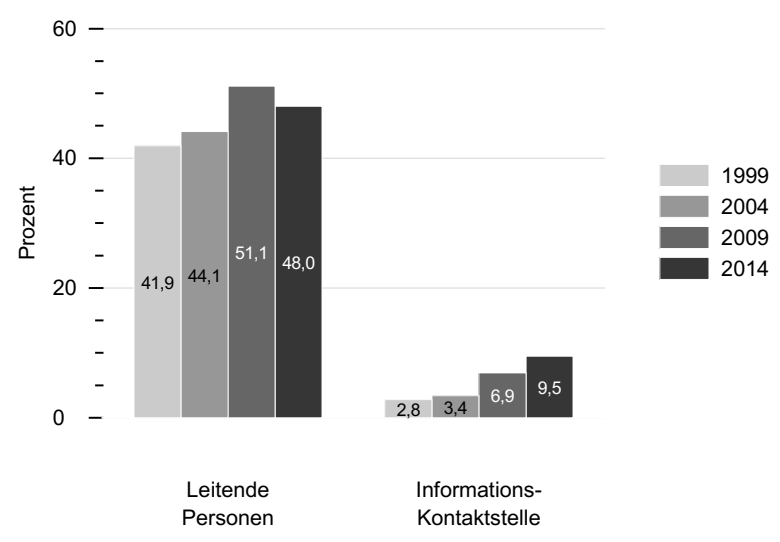

Quelle: FWS, gewichtet, eigene Berechnungen (DZA). Basis: Alle Engagierten. FWS 1999 ( $n=4.731)$, FWS 2004

$(n=5.142)$, FWS 2009 ( $n=7.023)$, FWS $2014(n=12.516)$.

\subsection{Motive für Engagement}

Insgesamt werden von den Engagierten vor allem Motive für ihre Tätigkeiten genannt, die sich auf das eigene Wohlbefinden oder auf soziale Aspekte beziehen (Abbildung 15-8). Mit 93,9 Prozent stimmen die meisten Personen im
Alter ab 14 Jahren der Aussage voll oder eher zu, dass ihr Engagement ihnen Spaß macht (davon 80,0 Prozent volle Zustimmung). Weiterhin will die überwiegende Mehrheit der Engagierten mit anderen Menschen zusammenkommen (82,0 
Prozent), die Gesellschaft mitgestalten (81,0 Prozent) und mit Menschen anderer Generationen zusammensein (80,1 Prozent).

Motive, die sich eher auf einen materiellen, beruflichen oder einen Statusgewinn durch das Engagement einer Person beziehen, werden weit weniger häufig genannt. So gibt zwar gut über die Hälfte der Wohnbevölkerung im Alter ab 14 Jahren an, durch ihr Engagement Qualifikationen erwerben zu wollen, die im Leben wichtig sind (51,5 Prozent). Aber nur noch 31,5 Prozent wollen Ansehen und Einfluss gewinnen und etwa ein Viertel hofft, durch das Engagement beruflich voranzukommen. Das am wenigsten wichtige Motiv ist das finanzielle: Insgesamt 7,2 Prozent geben an, durch ihr Engagement etwas dazuverdienen zu wollen. Wenn aber berücksichtigt wird, dass während des Interviews explizit nach Engagement gefragt wurde, für das die Befragten kein oder nur wenig Geld erhalten (siehe Beschreibung der Engagementabfrage in Kapitel 2), so erscheint der Prozentsatz der finanziell motivierten Engagierten doch überraschend zu sein. Dennoch bleibt es eine kleine Minderheit der Engagierten, für die der finanzielle Anreiz von Bedeutung ist. Zudem muss der Zusammenhang mit den anderen Motiven betrachtet werden, die für die meisten Engagierten eine wichtigere Rolle spielen. Sicherlich ist ein kleiner Beitrag zur Haushaltskasse für einige Engagierte nicht unwillkommen, solange die anderen Motive ebenfalls erfüllt werden, wie zum Beispiel Spaß zu haben oder mit Menschen zusammenzukommen.

\section{Abbildung 15-8: Angaben der freiwillig Engagierten zu den Motiven für ihr Engagement 2014}

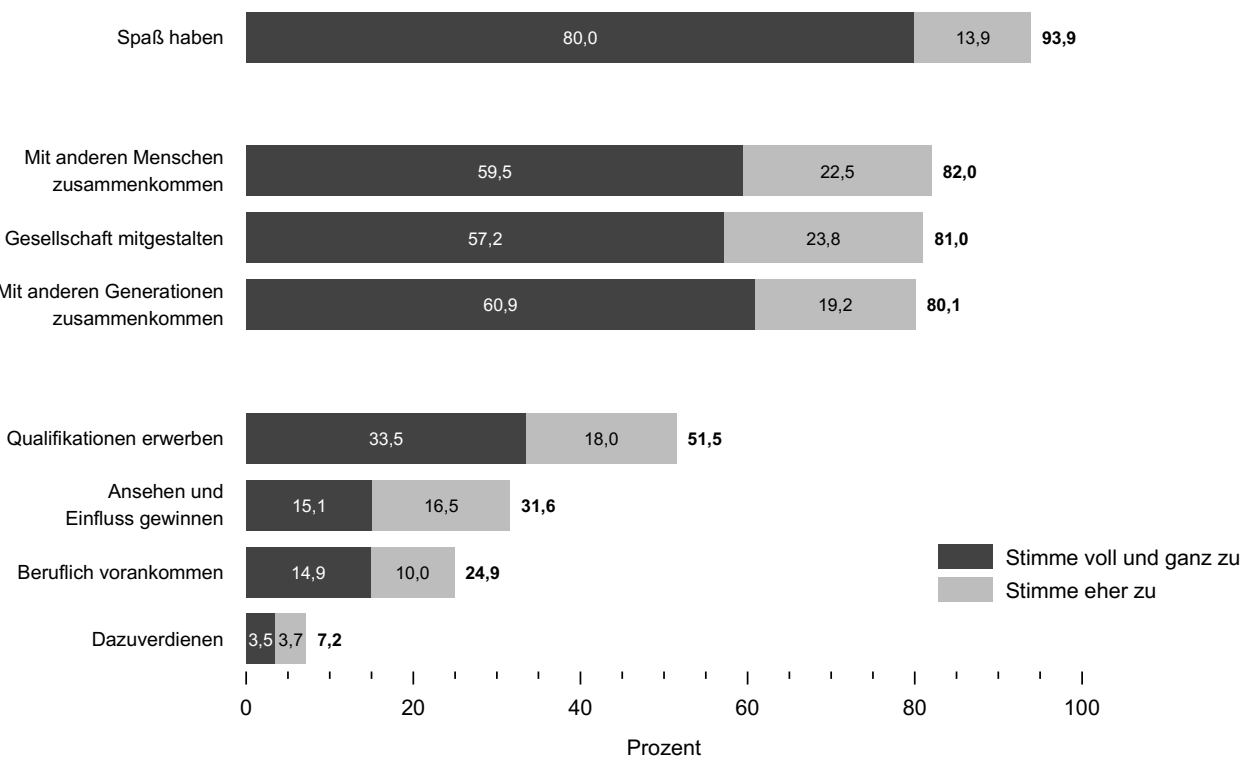

Quelle: FWS 2014, gewichtet, eigene Berechnungen (DZA). Basis: Alle Engagierten ( $n=11.651-12.506$ ).

Für die große Mehrheit der Engagierten steht also im Mittelpunkt, etwas zu tun, das ihnen Freude bereitet, dass sie etwas mit anderen Menschen zusammen tun können und dabei einen kleinen Beitrag für die Gesellschaft leisten können. Da- bei ist ihnen weniger wichtig, ihre Stellung in der Gesellschaft oder auf dem Arbeitsmarkt zu verbessern oder finanziell zu profitieren, auch wenn diese Motive trotzdem für viele eine - wenn auch untergeordnete - Rolle spielen. 
Im Geschlechtervergleich zeigt sich, dass Frauen anteilig häufiger als Männer Motive angeben, die sich auf das eigene Wohlbefinden oder soziale Aspekte beziehen, während Männer eher als Frauen Motive nennen, die einen materiellen oder immateriellen Gewinn beinhalten (Abbildung 15-9). In der Engagementbiografie von Frauen mag durch den anderen Zugang zum Engagement, der vor allem in den sozialen und in- formellen Bereich und seltener in Leitungspositionen führt (siehe Kapitel 11), der qualifizierende und finanzielle Aspekt eher in den Hintergrund treten. Auffällig ist jedoch, dass Frauen und Männer sich in Bezug auf die Reihenfolge der Wichtigkeit dieser Motive kaum unterscheiden: Spaß ist für beide Geschlechter am wichtigsten, Dazuverdienen am unwichtigsten. 
Abbildung 15-9: Angaben der freiwillig Engagierten zu den Motiven für ihr Engagement 2014, nach Geschlecht

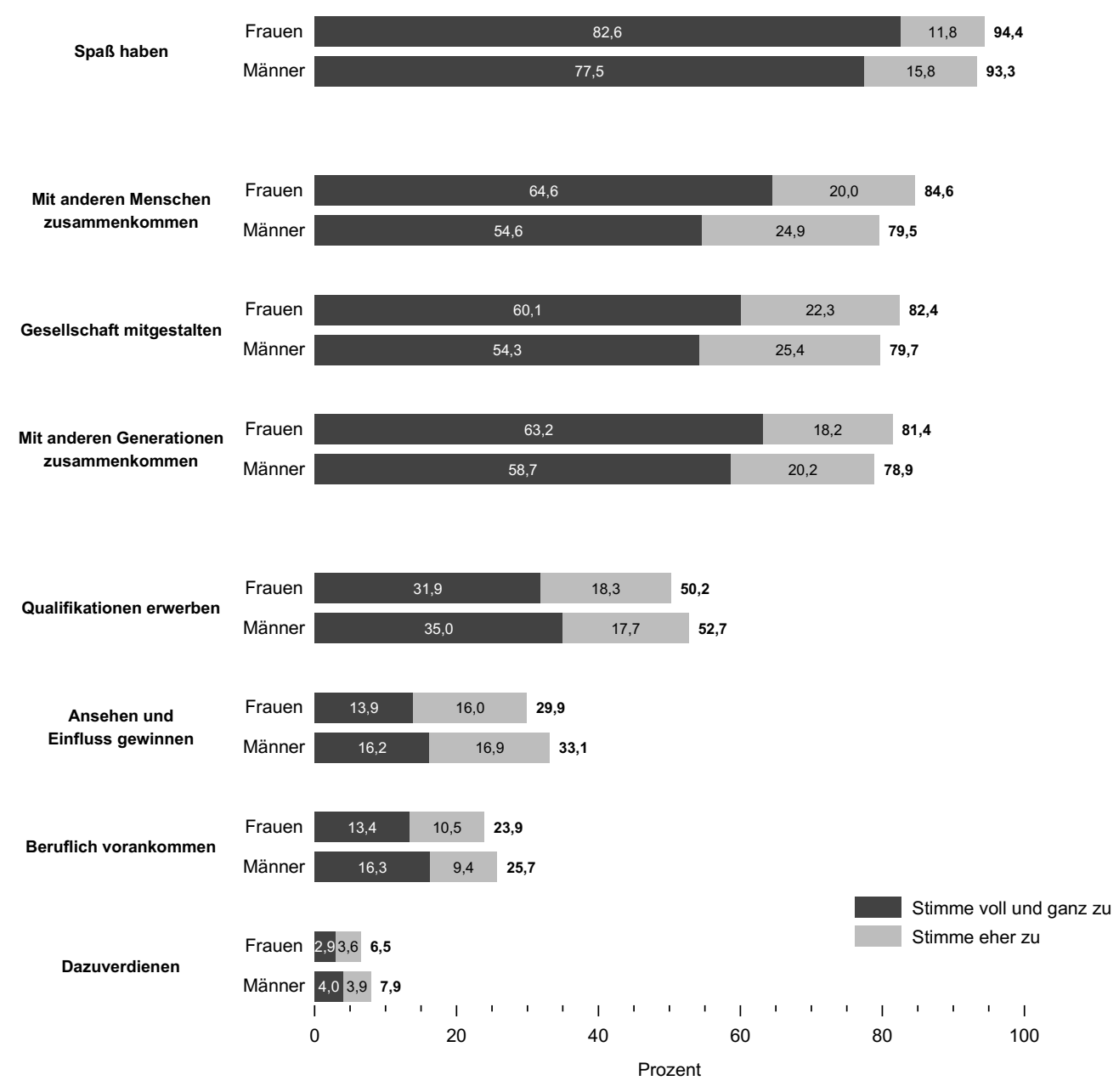

Quelle: FWS 2014, gewichtet, eigene Berechnungen (DZA). Basis: Alle Engagierten ( $n=11.651-12.506)$. 
Abbildung 15-10: Angaben der freiwillig Engagierten zu den Motiven für ihr Engagement 2014, nach Alter

Spaß haben

\section{Mit anderen Menschen zusammenkommen}

Gesellschaft mitgestalten

Mit anderen Generationen zusammenkommen

Qualifikationen erwerben

Ansehen und Einfluss gewinnen

Beruflich vorankommen

Dazuverdienen

Dazuverdienen

14-29 Jahre

30-49 Jahre

50-64 Jahre

65 Jahre und älter

14-29 Jahre

30-49 Jahre

50-64 Jahre

65 Jahre und älter

14-29 Jahre

30-49 Jahre

50-64 Jahre

65 Jahre und älter

14-29 Jahre

30-49 Jahre

50-64 Jahre

65 Jahre und älter

14-29 Jahre

30-49 Jahre

50-64 Jahre

65 Jahre und älter

14-29 Jahre

30-49 Jahre

50-64 Jahre

65 Jahre und älter

\begin{tabular}{|c|c|c|c|c|}
\hline \multicolumn{4}{|c|}{50,4} & 25,5 \\
\hline 33,4 & & & 9,5 & 52,9 \\
\hline 27,9 & & 14,6 & 42,5 & \\
\hline 19,5 & 10,3 & 29,8 & & \\
\hline
\end{tabular}

\begin{tabular}{|c|c|c|}
\hline 18,8 & \multicolumn{2}{|c|}{21,9} \\
\hline 13,0 & 15,7 & 28,7 \\
\hline 13,1 & 15,2 & 28,3 \\
\hline 17,2 & 12,9 & 30,1 \\
\hline
\end{tabular}

14-29 Jahre

30-49 Jahre

50-64 Jahre

65 Jahre und älter

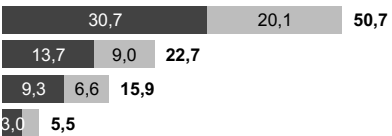
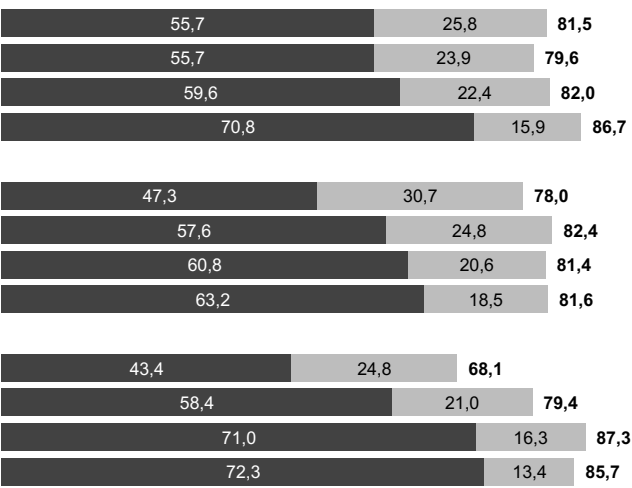

5,7

\section{5,0} 93,4

93,6
93,7 
Im Vergleich der Altersgruppen zeigen eher gewinnorientierte Motive eine andere Wichtigkeit. Diese Motive werden am ehesten von Personen der jüngsten Altersgruppe (14 bis 29 Jahre) genannt (Abbildung 15-10). Sie möchten gern ihr Engagement, das anderen zugutekommt, mit ihrer persönlichen Weiterentwicklung verbinden. Junge Menschen werden auch häufig durch den Hinweis auf diese Möglichkeiten für freiwilliges Engagement geworben. So kann das Freiwillige Soziale Jahr beispielsweise als ein Jahr verstanden werden, dass auch Orientierung für das weitere (Berufs-)Leben bietet. Auch gibt die jüngste Altersgruppe etwas häufiger als Ältere das Motiv ,Spaß' an, hier sind die Altersgruppenunterschiede aber sehr gering ausgeprägt. Soziale Motive werden jedoch eher von den älteren Altersgruppen im Vergleich zu den jüngeren angegeben. Die Gesellschaft mitgestalten wollen am ehesten 30 - bis 49-Jährige. Während es für die älteste Altersgruppe allgemein wichtig ist mit anderen Menschen zusammenzukommen, geben auch die 50- bis 64-Jährigen häufig an, dass sie der Austausch mit anderen Generationen motiviert. Hier wird deutlich, dass die Geselligkeit im höheren Alter wichtiger ist und in der Regel das eigene Ansehen, die Qualifikation und be- sonders finanzielle Leistungen als Motiv in den Hintergrund rücken. Dieses Ergebnis entspricht der allgemeinen Entwicklung im Alter, dass das soziale Netzwerk an Bedeutung gewinnt und das Interesse an neuen Erfahrungen angesichts der bereits gewonnenen Lebenserfahrung etwas geringer wird (Carstensen 1995).

Analog zu den oben beschriebenen jungen Menschen wird deutlich, dass nach Bildungsgruppen betrachtet vor allem Schülerinnen und Schüler die gewinnorientierten Motive angeben (Abbildung 15-11). Für die Gruppe der Schülerinnen und Schüler ist es eher wichtig, Ansehen und Einfluss zu gewinnen, beruflich voranzukommen, Qualifikationen zu erwerben sowie sich etwas dazu zu verdienen. Befragte mit niedriger Bildung geben im Vergleich zu den anderen Bildungsgruppen eher sozial-orientierte Motive an, nämlich mit anderen Menschen generell als auch mit Menschen anderer Generationen zusammensein $\mathrm{zu}$ wollen. Personen mit mittlerer und hoher Bildung geben etwas häufiger als andere Bildungsgruppen an, an ihrem Engagement Spaß zu haben; Spaß ist aber wiederum für alle unabhängig von der Bildung sehr wichtig. Die Gesellschaft mitzugestalten ist wichtiger für Engagierte mit höherer Bildung. 
Abbildung 15-11: Angaben der freiwillig Engagierten zu den Motiven für ihr Engagement 2014, nach Bildung

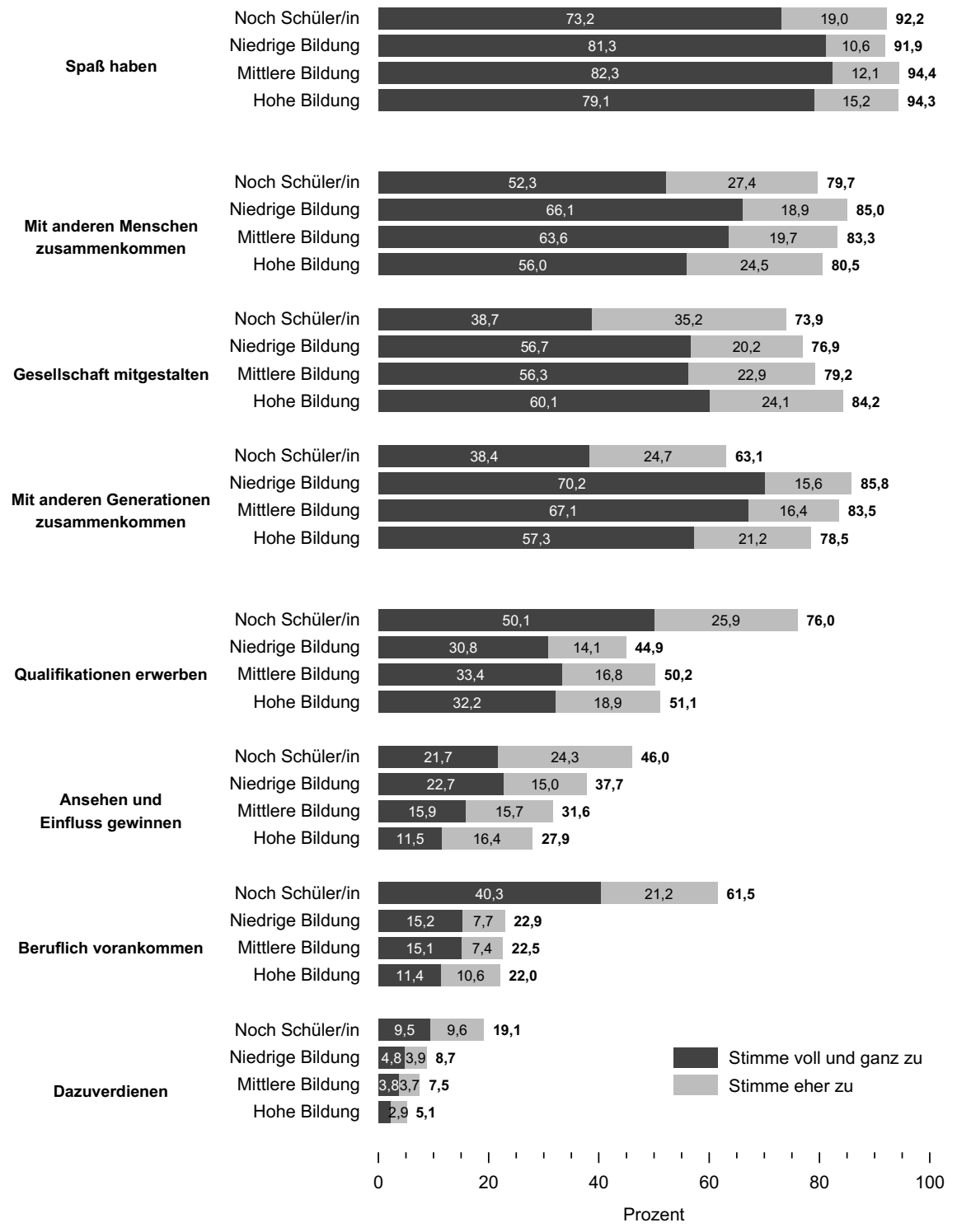

Quelle: FWS 2014, gewichtet, eigene Berechnungen (DZA). Basis: Alle Engagierten ( $n=11.647-12.502$ ). 


\subsection{Fazit}

Anstöße und Motive für freiwilliges Engagement hängen mit der persönlichen Situation der Engagierten zusammen. So sind für Engagierte in unterschiedlichen Lebensphasen jeweils verschiedene Themen von Bedeutung. Hierfür spielen neben dem Alter aber auch weitere Aspekte wie die Schulbildung und das Geschlecht eine Rolle. Jeweils etwa die Hälfte der Engagierten wurde aus eigener Initiative freiwillig tätig oder wurde gefragt. Die Eigeninitiative ist vermutlich am wichtigsten für jene freiwilligen Tätigkeiten, die außerhalb von klassischen Organisationsstrukturen eingebettet und daher informeller organisiert sind. Die im Vergleich zu den Vorwellen größere Wichtigkeit der Eigeninitiative könnte auch zum Teil die gestiegene Engagementquote erklären, sofern diese auf die vermehrte Verbreitung von eben jenen informelleren freiwilligen Tätigkeiten zurückgeht (siehe Kapitel 3). Dennoch bleibt es wichtig, dass Menschen direkt angesprochen und zum Engagement motiviert werden. Es zeigt sich, dass diese Art der Ansprache durchaus erfolgversprechend ist.

Hierbei können die Erkenntnisse zu Unterschieden in den Bevölkerungsgruppen hilfreich sein. Besonders Männer und Personen in der Altersgruppe der 30- bis 64-Jährigen engagieren sich, nachdem sie von leitenden Personen der Gruppe oder Organisation angesprochen wurden. Es ist denkbar, dass Männer stärker bereits in formalere Aktivitäten eingebunden sind, zum Beispiel im Sportverein, und daher häufiger gefragt werden. Frauen und Menschen der jüngsten und der ältesten Altersgruppe hingegen werden eher aus eigenem Antrieb heraus freiwillig tätig. Frauen engagieren sich beispielsweise häufiger für soziale Themen und auf informeller Basis (siehe Kapitel 3 und Kapitel 20). Hier gibt es möglicherweise mehr Gelegenheiten, sich aus eigener Initiative zu engagieren.

Der direkte Kontakt im Engagementumfeld sowie im persönlichen sozialen Umfeld sind die wichtigsten Anstöße zur Aufnahme eines Engagements. Vor allem Männer geben an, dass sie direkt durch Personen aus der Gruppe oder Organisation angesprochen wurden, während Frauen eher als Männer aufgrund von Erfahrungen in der eigenen Familie oder durch Informationen aus Kontaktstellen oder dem Internet zum Engagement gefunden haben. Hier gibt es vermutlich noch Potenzial auf Seiten der Organisationen, Frauen häufiger gezielt anzusprechen. Auch lohnt es sich offenbar, Kontakt- und Informationsstellen auszubauen, da seit dem Jahr 1999 immer mehr Engagierte berichteten, durch diese ihre freiwillige Tätigkeit gefunden zu haben.

Neben diesen Anstößen nennen Engagierte insgesamt am häufigsten Motive, die sich auf das eigene Wohlbefinden oder auf soziale Aspekte, wie die Interaktion mit anderen Menschen, beziehen. Eher gewinnorientierte Motive werden sowohl insgesamt als auch innerhalb der untersuchten gesellschaftlichen Gruppierungen weniger häufig genannt. Unabhängig von Geschlecht, Alter oder Bildung ist für alle Engagierten der Spaß bei ihrer freiwilligen Tätigkeit sehr wichtig. Er steht aber als Motiv fast nie allein, sondern tritt gemeinsam mit sozialen und auch gewinnorientierten Motiven auf. Kaum Bedeutung hingegen hat für die meisten der Engagierten, durch das Engagement Geld dazu zu verdienen. Dies bestätigt den Befund im Kapitel zu Geldzahlungen, Sachzuwendungen und Kostenerstattung im freiwilligen Engagement (siehe Kapitel 14), dass ein Trend zur Monetarisierung nicht zu erkennen ist. Freiwilliges Engagement spielt als Einkommensquelle also kaum eine Rolle.

Zwischen den verschiedenen gesellschaftlichen Gruppen lassen sich aber Differenzierungen erkennen: Soziale Aspekte sowie auf das eigene Wohlbefinden bezogene Motive werden häufiger von Frauen und von älteren Befragten genannt, während gewinnorientierte Motive eher von Männern sowie von jüngeren Personen beziehungsweise Schülerinnen und Schülern angegeben werden. Insgesamt hängen Motive also stark mit der Lebenssituation zusammen. 
Verschiedene Studien zu Geschlechterunterschieden im Engagement haben festgestellt, dass Frauen eher altruistische Motive berichten als Männer (Burns et al. 2008). Andererseits wurde aber auch diskutiert, dass die Qualifikation im Engagement vor allem für junge Menschen von Bedeutung ist (Gensicke 2015). Je nach Lebenssituation können sich die Unterschiede zwischen den Geschlechtern relativieren. Die Geschlechterunterschiede finden sich hinsichtlich der Ausrichtung der Motive ebenfalls in den freiwillig übernommenen Aufgaben und den Engagementbereichen wieder (siehe Kapitel 3). So sind Frauen eher in sozialen Bereichen und Männer eher in Bereichen mit den Themen Freizeit oder Politik freiwillig engagiert. Hierbei leisten Frauen etwas eher persönliche Hilfen, während Männer eher administrative und repräsentative Aufgaben übernehmen (Moschner 2002) und auch häufiger in Leitungsfunktionen zu finden sind (siehe Kapitel 11). Dies ist kongruent zu den Unterschieden zwischen Frauen und Männern hinsichtlich der Motive und möglicherweise dadurch erklärbar.

Deutlich haben sich bezüglich der Motive Unterschiede zwischen den Altersgruppen gezeigt. In der Literatur wird argumentiert, dass sich Motivationslagen mit zunehmendem Alter verändern (Oostlander, Güntert \& Wehner 2015). Ältere Menschen ziehen weniger Gewinn aus lern- und karriereorientierten Aktivitäten und fokussieren sich eher auf den Erhalt ihres Wohlbefindens (Okun \& Schultz 2003). Dies wird auch in den Ergebnissen des Freiwilligensurveys bezüglich der Motivation älterer Engagierter gespiegelt, die weniger gewinnorientiert ausfällt im Vergleich zu jüngeren Altersgruppen.

Hinsichtlich der Bildungsgruppen zeigt sich, dass der soziale, gesellige Aspekt zwar für alle Bildungsgruppen wichtig ist, dies gilt jedoch insbesondere für Niedriggebildete. Personen mit hoher Bildung geben hingegen häufiger als Mittel- oder Niedriggebildete an, dass sie die Gesellschaft mitgestalten wollen. Deutlich wird hier, dass Partizipation eng mit dem Bildungsgrad verbunden ist - höher gebildete Personen engagieren sich auch häufiger als andere (siehe Kapitel 3), auch weil sie eher glauben, damit etwas bewirken zu können (Böhnke \& Dathe 2010).

Diese Erkenntnisse sind besonders für die Frage von Bedeutung, wie Menschen zum Engagement und zu dessen Beibehaltung motiviert werden können. Die hier berichteten Gruppenunterschiede lassen darauf schließen, dass je nach Lebenssituation verschiedene Anstöße und Motive eine Rolle spielen. So könnten bei der Werbung für Engagement in den Medien oder über Informations- und Kontaktstellen die speziellen Motive der jeweiligen Zielgruppe berücksichtigt werden. Organisationen und Gruppen können zielgruppengerecht um neue Engagierte werben, die am ehesten an den persönlichen Entwicklungsmöglichkeiten der jeweiligen Tätigkeit interessiert sein könnten. Braun und Bischoff beschreiben beispielsweise Seniorenbüros, die über Angebote zur Selbsthilfe, Bildungs- und Informationsveranstaltungen gezielt ältere Menschen ansprechen und so niedrigschwellige Angebote schaffen, um sie für freiwillige Tätigkeiten zu interessieren. Sie sollen überzeugt werden, dass sie sich auch im höheren Alter einbringen können. Der für ältere Menschen wichtige soziale Aspekt freiwilligen Engagements wird zum Beispiel im Rahmen von ehrenamtlichen Besuchsdiensten in Krankenhäusern und Pflegeheimen relevant (Braun \& Bischoff 1999).

Der Einstieg in ein Engagement kann also durch direkte Ansprache, gut zugängliche Informationen und einfache Möglichkeiten zur Kontaktaufnahme erleichtert werden. Auch wäre es möglich, zielgruppenspezifisch auf die positiven Aspekte und Vorteile des Engagements hinzuweisen (Grano, Lucidi, Zelli \& Violani 2008). Personen mit niedriger Bildung könnte man beispielsweise gewinnen, indem Organisationen zeigen, dass sie während ihrer Tätigkeit mit anderen Menschen zusammentreffen, dass man sich dort austauschen und vielleicht sogar Freundschaften schließen kann. 


\section{Literatur}

Bierhoff, H.-W., Schülken, T., \& Hoof, M. (2007). Skalen der Einstellungsstruktur ehrenamtlicher Helfer (SEEH). Zeitschrift für Personalpsychologie, 6(1), 12-27.

Böhnke, P., \& Dathe, D. (2010). Rückzug der Armen. Der Umfang freiwilligen Engagements hängt von der materiellen Lage ab - und von Bildung. WZB Mitteilungen, 2010(128), 14-17.

Braun, J., \& Bischoff, S. (1999). Bürgerschaftliches Engagement älterer Menschen: Motive und Aktivitäten. Engagementförderung in Kommunen - Paradigmenwechsel in der offenen Altenarbeit. Berlin: Bundesministerium für Familie, Senioren, Frauen und Jugend.

Burns, D. J., Reid, J., Toncar, M., Anderson, C., \& Wells, C. (2008). The Effect of Gender on the Motivation of Members of Generation Y College Students to Volunteer. Journal of Nonprofit \& Public Sector Marketing, 19(1), 99-118.

Carstensen, L. L. (1995). Evidence for a Life-Span Theory of Socioemotional Selectivity. Current Directions in Psychological Science, 4(5), 151-156.

Clary, E. G., Snyder, M., Ridge, R. D., Copeland, J., Stukas, A. A., Haugen, J., \& Miene, P. (1998). Understanding and Assessing the Motivations of Volunteers. A Functional Approach. Journal of Personality and Social Psychology, 74(6), 1516-1530.

Deutscher Bundestag (Hrsg.) (2012). Erster Engagementbericht - Für eine Kultur der Mitverantwortung. Bericht der Sachverständigenkommission und Stellungnahme der Bundesregierung (Bundestagsdrucksache 17/10580). Berlin: Deutscher Bundestag.

Gensicke, T. (2015). Freiwilliges Engagement in Deutschland: Freiwilligensurvey 2009. Wiesbaden: Springer VS.

Grano, C., Lucidi, F., Zelli, A., \& Violani, C. (2008). Motives and determinants of volunteering in older adults: An integrated model. The International Journal of Aging \& Human Development, 67(4), 305-326.

Hacket, A., \& Mutz, G. (2002). Empirische Befunde zum bürgerschaftlichen Engagement. Aus Politik und Zeitgeschichte, 52(9), 39-46.

Haski-Leventhal, D., Ronel, N., York, A. S., \& Ben-David, B. M. (2008). Youth volunteering for youth: Who are they serving? How are they being served? Children and Youth Services Review, 30(7), 834-846.

Haumann, W. (2014). Motive des bürgerschaftlichen Engagements. Kernergebnisse einer bevölkerungsrepräsentativen Befragung durch das Institut für Demoskopie Allensbach im August 2013. Berlin: Bundesministerium für Familie, Senioren, Frauen und Jugend.

Moschner, B. (2002). Altruismus und Egoismus. Was motiviert zum Ehrenamt? (Diskussionspapier 20). Bielefeld: Universität Bielefeld.

Okun, M. A., \& Schultz, A. (2003). Age and Motives for Volunteering: Testing Hypotheses Derived From Socioemotional Selectivity Theory. Psychology and Aging, 18(2), 231-239.

Oostlander, J., Güntert, S. T., \& Wehner, T. (2015). Motive für Freiwilligenarbeit - der funktionale Ansatz am Beispiel eines generationenübergreifenden Projekts. In: T. Wehner \& S. T. Güntert (Hrsg.) Psychologie der Freiwilligenarbeit (S. 59-76). Berlin: Springer.

Wilson, J., \& Musick, M. (1998). The Contribution of Social Resources to Volunteering. Social Science Quarterly, 79(4), 799-814.

Winker, G., \& Degele, N. (2009). Intersektionalität. Bielefeld: transcript.

Zimmer, A., \& Backhaus-Maul, H. (2012). Engagementförderung vor Ort - Was gilt es in den Blick zu nehmen? Eine Arbeitshilfe für lokale Entscheidungsträger. Münster: Westfälische Wilhelms-Universität Münster.

Zimmer, A., \& Rauschenbach, T. (2011). Bürgerschaftliches Engagement unter Druck? Einleitung. In: T. Rauschenbach \& A. Zimmer (Hrsg.) Bürgerschaftliches Engagement unter Druck? Analysen und Befunde aus den Bereichen Soziales, Sport und Kultur (S. 11-28). Opladen: Barbara Budrich.

Open Access Dieses Kapitel wird unter der Creative Commons Namensnennung 4.0 International Lizenz (http://creativecommons.org/licenses/by/4.0/deed.de) veröffentlicht, welche die Nutzung, Vervielfältigung, Bearbeitung, Verbreitung und Wiedergabe in jeglichem Medium und Format erlaubt, sofern Sie den/die ursprünglichen Autor(en) und die Quelle ordnungsgemäß nennen, einen Link zur Creative Commons Lizenz beifügen und angeben, ob Änderungen vorgenommen wurden.

Die in diesem Kapitel enthaltenen Bilder und sonstiges Drittmaterial unterliegen ebenfalls der genannten Creative Commons Lizenz, sofern sich aus der Abbildungslegende nichts anderes ergibt. Sofern das betreffende Material nicht unter der genannten Creative Commons Lizenz steht und die betreffende Handlung nicht nach gesetzlichen Vorschriften erlaubt ist, ist für die oben aufgeführten Weiterverwendungen des Materials die Einwilligung des jeweiligen Rechteinhabers einzuholen. 